\title{
Constraints on the Yields of the First Supernovae in the Universe
}

\author{
Roger Cayrel* \\ $\dagger$ \\ Observatoire de Paris 61 Av. de l'Observatoire 75014 Paris, France \\ E-mail: Roger.Cayrel@obspm. fr
}

The study of the chemical composition of the most primitive stars of the galactic halo has been made possible with the help of large surveys aimed at finding such stars, and by powerful new instruments, as the Keck telescopes, the Subaru telescope, and the ESO Very Large Telescope. The atmospheres of these primitive stars possess, per hydrogen atom, from 1/1000th to 1/10000th less supernovae-made elements than the Sun, and reflect the yields of the first supernovae. It was once expected that these yields would show a larger scatter than those in the more metal-rich Population II stars, which have been enriched by many more supernovae explosions than the earlier generations. If we leave aside one class of objects, the Carbon-Enhanced Metal-Poor (CEMP) stars, which is the topic of another talk at this conference, a rather well-defined set of abundance ratios emerge for $\mathrm{C}$ to $\mathrm{Zn}$ amongst the most primitive population, with a scatter that is surprisingly small. The quality of the high-resolution spectroscopic data is such that the observed level of scatter in the measured elemental abundances for these species is no longer limited by accuracy of the observations, nor by other errors inherent to the analysis of the data. By way of contrast, amongst the neutron-capture elements produced by the $r$-process, at a given metallicity a spread reaching a factor of over 1000 exists for elements such as $\mathrm{Ba}$. The stable portion of the $r$-process pattern observed in such stars is the second peak $(\mathrm{Z}=56$ to 72$)$, in which the relative abundances of these elements in very metal-poor stars are almost indistinguishable from their inferred proportions in solar-system material.

Recent observations have permitted the determination of the abundances of uranium, thorium, and lead produced by the $r$-process in extremely metal-poor stars, and indicate that lead is mainly produced by radioactive decay of the actinides (as opposed to other direct channels). In addition, the observed U/Th ratio has been shown to be the best available radioactive cosmic chronometer, on timescales of interest to cosmology.

International Symposium on Nuclear Astrophysics - Nuclei in the Cosmos - IX

CERN

\section{* Speaker.}

${ }^{\dagger}$ Part of this talk is based on observations of the Large Program 165.N-0276(A) obtained with the Very Large Telescope of the European Southern Observatory at Paranal, Chile 


\section{Introduction}

The first supernovae have left present-day astronomers a "smoking gun", in the sense that their ejecta have been captured in the atmospheres of the oldest stars that are still observable. Up until the mid 1990s ([1],[2]), astrophysicists knew too few such very old stars to perform analyses of this valuable diagnostic. Thanks to the first major search for very metal-poor stars, referred to as either the HK survey, or the BPS survey, from the name of the authors (Beers, Preston, \& Shectman, [3]), the number of stars known with iron abundances lower than those of the Milky Way globular clusters has jumped from a handful to more than several hundred, making possible a detailed analysis of a significant sample of such stars. Performing such analyses was, however, quite difficult, even with the $4-5 \mathrm{~m}$ class telescopes available at the time, let alone with a $2.5 \mathrm{~m}$ telescope as was used by McWilliam et al. in the seminal work cited above. The construction of $8 \mathrm{~m}$ to $10 \mathrm{~m}$ telescopes with efficient optical/near-UV spectrographs has led to a dramatic change in the situation, enabling abundance ratios to be measured with accuracies at least four times better than previously possible. In the following review we report a few of the recent determinations of abundance ratios for species of greatest interest to nuclear astrophysics.

A remarkable opportunity now exists for the determination of $r$-process yields by early supernovae, as a number of very and extremely metal-poor stars have been discovered that exhibit very large enhancements of their neutron-capture $r$-elements. It is now possible to consider the origin of the $r$-process elements quite independent of the need to decompose the solar mixture into $r$-and $s$ process contributions; a major step forward for nuclear astrophysics. One such star, CS 22892-052, has already been the subject of numerous papers. A comprehensive study of this star by [4] supplies important observational constraints on the nucleosynthesis of the neutron-capture $r$-elements. A second star, CS 31082-001, also an extremely metal-poor giant, has brought new constraints on the origin of the actinides, and on the production channels for $r$-process lead. A section below is devoted to this topic.

\section{Constraints on Big Bang Nucleosynthesis}

Following the recent results from the Wilkinson Microwave Anisotropy Probe (WMAP) [5], predictions of primordial nucleosynthesis has become a zero free-parameter game [6]. Astrophysical constraints on primordial nucleosynthesis come from the measured deuterium/hydrogen ratio in high-redshift matter, from the $\mathrm{He} / \mathrm{H}$ ratio in matter with small amounts of astration (found in a few blue compact galaxies), and from the ${ }^{7} \mathrm{Li} / \mathrm{H}$ ratio measured in unevolved halo stars with very low astration (in practice very metal-poor subdwarfs or main-sequence turn-off stars). The first two items support well the theoretical prediction of Big Bang Nucleosynthesis (BBN), whereas the ${ }^{7} \mathrm{Li}$ observations fit in order of magnitude only; the observed ${ }^{7} \mathrm{Li} / \mathrm{H}$ (in number of atoms) being lower by 0.4 dex than the BBN predicted value of $10^{-9.36 \pm 0.03}$. Several stellar depletion mechanisms exist that could have altered a higher initial abundance, but the subject is difficult and the simplest explanation of a plateau is of course - no depletion! The role of diffusion has been thoroughly studied [7], and further work is in progress. 


\section{Constraints on Early Alpha- and Iron-Peak Element Production}

The content of this section is mostly a summary of the two papers "First Stars V" [8], and "First Stars VI" [9], which reported results from the ESO-VLT large programme "First Stars." Table 1 lists the essential results. The "First Stars" program had 14 participants, from six different countries (France, Italy, Denmark, the USA, Sweden, and Brazil). The First Stars programme carried out a detailed analysis of about 60 very metal-poor stars from the HK survey, with an unprecedented signal/noise ratio. For most of the program stars we achieved a $\mathrm{S} / \mathrm{N}$ ratio of $100-200$ per pixel, with six pixels per resolution element. There are two highlights of this program. For stars below $[\mathrm{Fe} / \mathrm{H}]^{1}=-3.0$, the observed trends of most elemental species with metallicity have vanished, and rather well-defined abundance ratios are obtained. The expectation is that these ratios are those that have arisen from zero-metallity or (at least) extremely low-metallicity $\mathrm{SNe}$ ejecta. These ratios are listed in Table 1.

Previous theoretical work [10] indicated that a classical SN II explosion pollutes pristine material of about $10^{5}$ solar masses with its ejecta. Taking, for example, the theoretical yields of [11], this roughly corresponds to the level of the abundances observed in the stars with $[\mathrm{Fe} / \mathrm{H}] \leq-3.0$ (the Extremely Metal-Poor, or EMP) stars. It is thus tempting to associate the abundances observed in the EMP stars with either the elemental yields arising from a single SN II explosion, or just a few such explosions. Here comes the surprise: the scatter in the observed abundance ratios is almost undetectable, according to Table 1, and as shown in Figures 1-5. The scatter that might have been expected due to the contributions from individual SNe of different masses is not observed! This conclusion is further strengthened by [12], in which the scatter on the derived $\mathrm{Mg}$ abundances in their sample due to errors in effective temperatures and gravity determinations is minimized by using a sample of stars with almost identical temperatures and gravities. The scatter on $[\mathrm{Mg} / \mathrm{Fe}]$ drops to $0.06 \mathrm{dex}$, even considering random observational errors, estimated to be on the order of 0.08 dex. The explanation of such an undetectable intrinsic scatter is highly surprising. It suggests that the ejecta of a rather large number of SNe have been mixed, before the diluted ejectas were able to form new stars of mass below $0.9 M_{\odot}$, i.e., those still shining today. If the ejecta of the first SN II were not trapped in their "minihalo cocoons", it might be the case that intergalactic space was polluted on very large scales before the birth of the now-observed EMP stars. This would also explain the uniformity of metallicity in globular clusters, born from already well-mixed matter.

\section{The Neutron-Capture $r$-process Elements}

Space limitations force us to skip the very exciting subject of the "lead" stars, except to note that it appears clear that Asymptotic Giant Branch stars were able to produce enormous amounts of lead in at least one of our very metal- poor stars, (CS 29497-030; [Fe/H] $=-2.8)$, in spite of their presumed longer evolutionary time scales (see [13]).

At low metallicities, there exists a striking departure in the behaviour of the elements from $\mathrm{C}$ to $\mathrm{Zn}$ with that of heavier elements. For example, at a given metallicity, the observed scatter in $\mathrm{Ba}$ is three orders of magnitude, while that of $\mathrm{Mg}$ is below $0.06 \mathrm{dex}$ ! The inescapable conclusion is that that the producion of $\mathrm{Mg}$ (or $\mathrm{Fe}$ ) and that of $\mathrm{Ba}$ are almost uncorrelated. Either $\mathrm{Ba}$ is not

\footnotetext{
${ }^{1}$ In this paper we use the symbol "[X/Y]" for $\log (X / Y)_{\text {star }}-\log [X / Y)_{\odot}$, where $\mathrm{X} / \mathrm{Y}$ is an abundance ratio.
} 


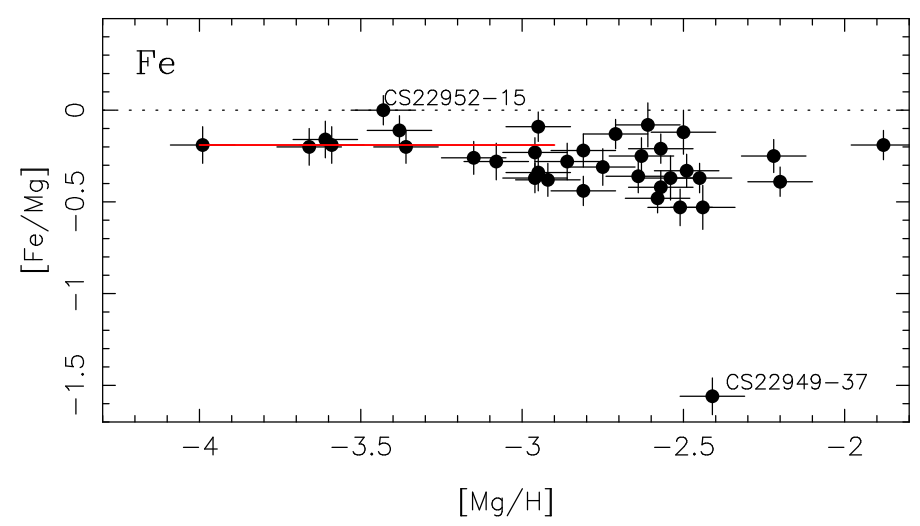

Figure 1: The observed $[\mathrm{Fe} / \mathrm{Mg}]$ ratio for the 14 most metal-poor stars in the sample considered, as a function of $[\mathrm{Mg} / \mathrm{H}]$.

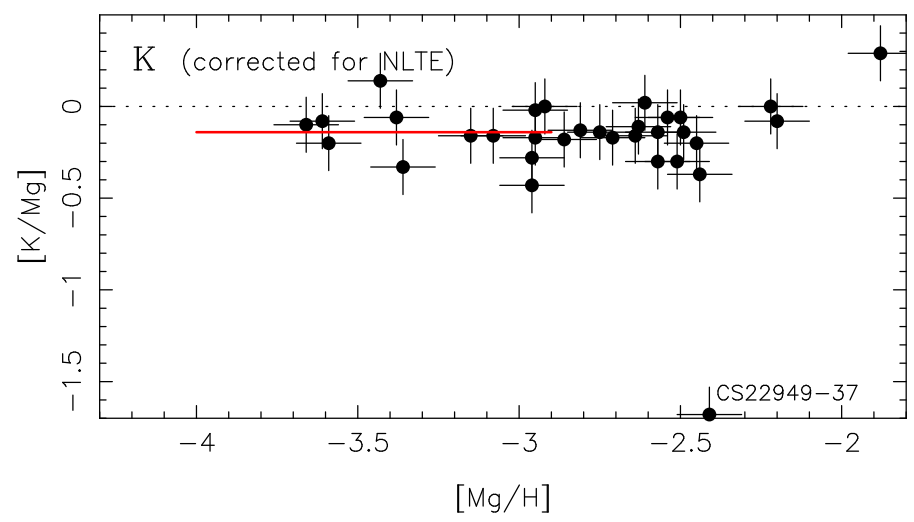

Figure 2: The observed $[\mathrm{K} / \mathrm{Mg}]$ ratio for the 14 most metal-poor stars in the sample considered, as a function of $[\mathrm{Mg} / \mathrm{H}]$.

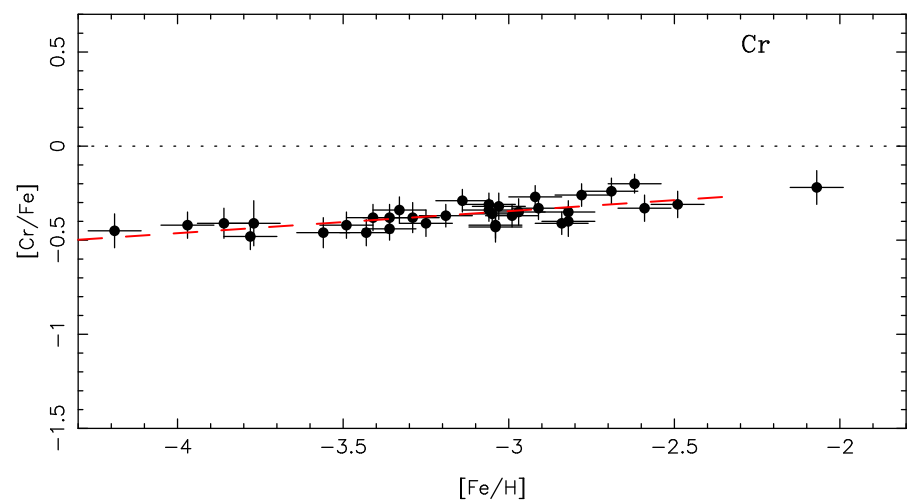

Figure 3: $[\mathrm{Cr} / \mathrm{Fe}]$ vs. $[\mathrm{Fe} / \mathrm{H}]$ in the full sample of 35 very metal-poor giants. Note the extremely small scatter, and the vanishing of the negative slope below $[\mathrm{Fe} / \mathrm{H}]=-3.0$. 


\begin{tabular}{l|c|c|c}
\hline \hline Element & Abundance & RMS Scatter & Number of Stars \\
\hline$[\mathrm{C} / \mathrm{Mg}]$ & -0.01 & 0.10 & 9 \\
{$[\mathrm{~N} / \mathrm{Mg}]$} & +0.00 & 0.11 & 9 \\
{$[\mathrm{O} / \mathrm{Mg}]$} & +0.32 & 0.21 & 13 \\
{$[\mathrm{Na} / \mathrm{Mg}]$} & -0.45 & 0.16 & 9 \\
{$[\mathrm{Al} / \mathrm{Mg}]$} & -0.39 & 0.05 & 9 \\
{$[\mathrm{Si} / \mathrm{Mg}]$} & +0.21 & 0.14 & 14 \\
{$[\mathrm{~K} / \mathrm{Mg}]$} & -0.14 & 0.14 & 13 \\
{$[\mathrm{Ca} / \mathrm{Mg}]$} & +0.06 & 0.09 & 14 \\
{$[\mathrm{Sc} / \mathrm{Mg}]$} & -0.17 & 0.14 & 14 \\
{$[\mathrm{Ti} / \mathrm{Mg}]$} & -0.01 & 0.09 & 14 \\
{$[\mathrm{Cr} / \mathrm{Mg}]$} & -0.63 & 0.09 & 14 \\
{$[\mathrm{Mn} / \mathrm{Mg}]$} & -0.65 & 0.20 & 14 \\
{$[\mathrm{Fe} / \mathrm{Mg}]$} & -0.21 & 0.10 & 14 \\
{$[\mathrm{Co} / \mathrm{Mg}]$} & +0.13 & 0.17 & 14 \\
{$[\mathrm{Ni} / \mathrm{Mg}]$} & -0.23 & 0.13 & 14 \\
{$[\mathrm{Zn} / \mathrm{Mg}]$} & +0.21 & 0.19 & 14 \\
\hline
\end{tabular}

Table 1: Abundances, relative to $\mathrm{Mg}$, of the elements from $\mathrm{C}$ to $\mathrm{Zn}$ in non- CEMP stars. The sample consists of halo giants with $[\mathrm{Mg} / \mathrm{H}]$ less than -3.0 . The last column is the number of objects considered.

formed in SN II explosions, or only in a very small subset of them. The four peaks of the $r$-process elements exhibit slightly different behaviours. The second peak has very stable abundance ratios; EMP stars exhibit the same patterns as those of solar-system $r$-process elements. However, the ratio of the first peak (e.g., Sr, Y, Zr) to the second-peak appears to exhibit variations from star to star. The actinides may have abundance ratios with the second peak varying too ([14],[15],[16]).

\section{The Actinides, Lead, and Cosmochronology}

\subsection{Thorium and Uranium}

The very metal-poor halo giant CS 22892-052 is noteworthy in presenting an overabundance, with respect to iron, of a factor of 50 for the neutron-capture elements produced by the $r$-process. Another, even more interesting star, was discovered during the "First Stars" program [17], CS 31082001, presenting a similar overabundance of the rare-earths, but an even larger overabundance of the actinides. This large overabundance (and the low carbon abundance in CS 31082-001, which is important because molecular carbon features can blanket the abundance signatures of the actinides) allowed the measurement of uranium in this star, as well as an accurate determination of the U/Th ratio. This ratio is considered as the most reliable cosmochronometer ([18],[19]), because the nuclei of Th and $\mathrm{U}$ are very similar, and are expected to be produced under identical astrophysical conditions, which is not necessarily the case, for example, for other cosmo-chronometer pairs such as Th and Eu.

Here we reverse the usual procedure, which is aimed at finding an age limit on the object(s) responsible for the formation of the actinides in this star, by adopting a theoretical value for the 
production ratio of $(\mathrm{U} / \mathrm{Th})_{0}$. The logic is straightforward. The age of the likely progenitors to this EMP star is already strongly constrained by cosmology and by independent estimates of the age of the globular clusters. Thus, instead, we use the observations to determine the production ratio (U/Th) $)_{0}$, a physical quantity of enormous interest to nuclear astrophysics. From the half-lives of ${ }^{232} \mathrm{Th}(14.05 \mathrm{Gyr})$, and ${ }^{238} \mathrm{U}$ (4.47 Gyr), it is easy to predict the abundance of U/Th which should be observed after a time $\Delta t$ Gyr:

$$
\log \left((\mathrm{U} / \mathrm{Th})_{\text {now }}\right)=\log \left((\mathrm{U} / \mathrm{Th})_{0}\right)-0.04596 \Delta t
$$

We estimate from Figure 4 that:

$$
\log \left(\mathrm{U}_{\text {now }}\right)=-1.92 \pm 0.10
$$

and from:

$$
\log \left(\mathrm{Th}_{\text {now }}\right)=-0.98 \pm 0.06
$$

that:

$$
\log \left((\mathrm{U} / \mathrm{Th})_{\text {now }}=-0.94 \pm 0.11\right.
$$

Based on the WMAP third-year data results (first reionization not before $\mathrm{z}=20$, i.e., an age of the Universe of $13.6 \mathrm{Gyr})$, and from the reasonable assumption that CS 31082-001 is older than the minimum age for galactic globular clusters $(\approx 12 \mathrm{Gyr})$, which are more metal-rich by factors of three, the following bracket is derived for $\Delta t$ :

$$
12.0 \mathrm{Gyr}<\Delta t<13.6 \mathrm{Gyr}
$$

This brackets $\log \left((\mathrm{U} / \mathrm{Th})_{0}\right.$ to be between:

$$
-0.498<\log \left((\mathrm{U} / \mathrm{Th})_{0}<-.205\right.
$$

or:

$$
0.317<(\mathrm{U} / \mathrm{Th})_{0}<0.624
$$

The estimates of this production ratio delivered by nuclear physicists are indeed in line with this astrophysical constraint, but tend to be closer to the upper limit. Observations of additional very and extremely metal-poor stars with detectable $\mathrm{U}$ and Th will help shrink the allowed range of this astrophysical constraint.

\subsection{Lead}

If one adopts a mean progenitor age of $12.8 \mathrm{Gyr}$, following the logic above, it is straightforward to estimate the amount of lead produced by the decay of the original amounts of ${ }^{232} \mathrm{Th}$ and ${ }^{238} \mathrm{U}$. This prediction is interesting to compare with the total lead abundance obtained by [20]. From:

$$
\varepsilon\left({ }^{206} \mathrm{~Pb}\right)=\varepsilon\left({ }^{238} \mathrm{U}_{\text {now }}\right) \times\left(2^{\Delta t / \tau}-1\right)
$$


where $\varepsilon$ is the abundance of the element with respect to hydrogen (on a scale where $\mathrm{n}(\mathrm{H})=10^{12}$ ), and taking $\tau$ as the half-life of the radioactive element, one obtains the amount of ${ }^{206} \mathrm{~Pb}$ expected from the decay of uranium. Similarly, with $\tau$ replaced by the half-life of ${ }^{232} \mathrm{Th}$, one obtains the amount of ${ }^{208} \mathrm{~Pb}$ produced from the decay of thorium. The total of the isotopes ${ }^{206} \mathrm{~Pb}$ and ${ }^{208} \mathrm{~Pb}$ is then $\log (\varepsilon(\mathrm{Pb}))=-0.77$. To this total one should add the ${ }^{207} \mathrm{~Pb}$ coming from the decay of ${ }^{235} \mathrm{U}$. Unfortunately, we cannot use the same method as above, since the remaining ${ }^{235} \mathrm{U}$ today is too small, due to its short half-life. Based on reports in the literature, it appears that this additional contribution is on the order of $80 \%$ of that coming from ${ }^{238} \mathrm{U}$, thus increasing the lead abundance predicted to arise from the decay of the actinides to $\log (\varepsilon(\mathrm{Pb}))=-0.64$.

Our measurement of total lead abundance in CS 31082-001 is $\log (\varepsilon(\mathrm{Pb}))=-0.55 \pm 0.15$ [20]. See Figure 5. Thus, the lead coming from decay of the actinides represents $85 \%$ of the total amount of the observed lead in this star. This is not in line with several papers that have based their estimates on a higher estimate of the $r$-process lead fraction in the Solar System. This is the first time that an abundance of $\mathrm{Pb}$ has been directly measured in a pure $r$-process yield, and the result is very low compared to expectation. Presumably, nature is telling us that our understanding of the mechanisms involved with lead production is still incomplete.

\section{Conclusions}

At present, roughly one hundred stars with an inferred very low pollution by $\mathrm{SNe}$ ejecta (of the order of only a few to $10 \mathrm{ppm}$ in mass, excluding helium) have been studied at high spectral resolution. It is fair to say that the bulk of these stars exhibit rather well-defined nuclear compositions, with star-to-star scatter in the alpha- and iron-peak elements that are surprisingly small. It is surely noteworthy that no star has been found less that is less polluted than the level expected to arise from the first supernovae, the lowest iron abundances recorded to date being for the Hyper Metal-Poor stars HE 0107-5240 $([\mathrm{Fe} / \mathrm{H}]=-5.3,[21])$ and $\mathrm{HE} \mathrm{1327-2326}([\mathrm{Fe} / \mathrm{H}]=-5.6,[22])$. It seems highly likely that matter that is less-polluted than this level was unable (for lack of efficient radiative processes) to fragment into stars less than the mass limit of the oldest main-sequence stars, presently about 0.9 solar mass. Theoretical work suggests much higher minimum masses, on the order of 100 solar masses, were probably the dominant contributors in the very early Galaxy. So, all the first stars have ended as $\mathrm{SNe}$, and the best we can do, and are indeed doing at present, is to observe stars that were formed when the pollution level of the cosmic gas reached a critical value.

\section{Thanks}

I am greatly indebted to T.C. Beers for assisting with the presentation of this review, and for the energy he has put in transforming the field of metal-poor stars, into the realm of the very metalpoor, extremely metal-poor, ultra metal-poor, and hyper metal-poor stars. Someday, soon, we hope to reach the long sought mega metal-poor stars! 


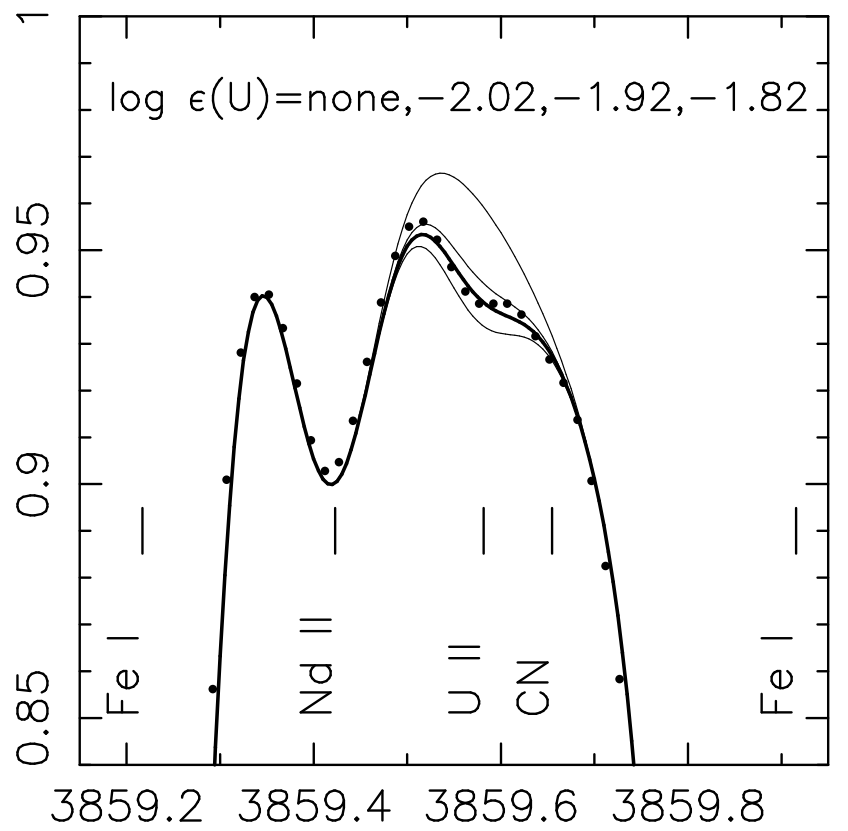

$\lambda(\AA)$

Figure 4: The uranium line at $3859 \AA$ in the star CS 30082-001.

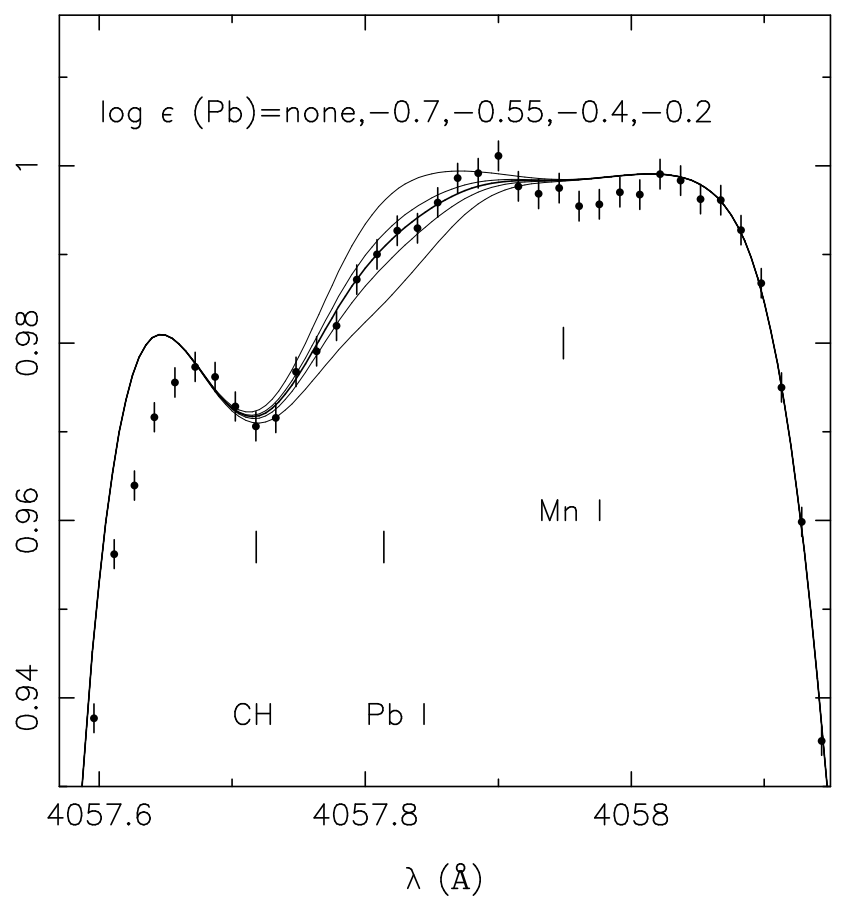

Figure 5: The lead line at $4057.8 \AA$ in CS 31082-001. 


\section{References}

[1] McWilliam, A. et al. 1995, Spectrocopic Analysis of 33 of the Most Metal Poor Stars. II., AJ, 109, 2757

[2] Norris, J.E., Ryan, S.G., \& Beers, T.C. 1996, Extremely Metal-poor Stars. II. Elemental Abundances and the Early Chemical Enrichment of the Galaxy, ApJ, 471, 254

[3] Beers, T.C., Preston, G.W., \& Shectman, S.A. 1992, A Search for Stars of Very Low Metal Abundance. II., AJ, 103, 1987

[4] Sneden, C., et al. 2003, The Extremely Metal-Poor, Neutron-Capture Rich Star CS 22892-052: A Comprehensive Abundance Analysis, ApJ, 591, 936

[5] see site http://lambda.gsfc.nasa.gov

[6] Coc, A. \& Vangioni, E. 2005, in IAU Symp. 228, From Lithium to Uranium: Elemental Tracers of the Early Cosmic Evolution, eds. V.Hill, P. François \& F. Primas, Li and Big-Bang Nucleosynthesis, p. 13

[7] Richard, O., Michaud, G., \& Richer, J. 2005, Implications of WMAP Observations on Li Abundance and Stellar Evolution Models, ApJ, 619, 538

[8] Cayrel, R., et al. 2004, First Stars V - Abundance Patterns from C to Zn and Supernovae Yields in the Early Galaxy, A \& A,416, 1117

[9] Spite, M., et al. 2005, First Stars VI - Abundances of C, N,O,Li and Mixing in Extremely Metal-Poor Giants. Galactic Evolution of the Light Elements, $A$ \& A, 430, 655

[10] Cioffi, D.F., McKee, C.F., \& Bertschinger, E. 1988, Dynamics of Radiative Supernova Remnants, ApJ, 334, 252

[11] Woosley, S.E., \& Weaver, T.A. 1995, The Evolution and Explosion of Massive Stars. II. Explosive Hydrodynamics and Nucleosynthesis, ApJS, 101, 181

[12] Arnone, E., et al. 2005, Mg Abundances in Metal-Poor Halo Stars as a Tracer of Early Galactic Mixing, $A \& A, \mathbf{4 3 0}, 507$

[13] Sivarani, T., et al. 2004, First Stars IV - CS 29497-030: Evidence for Operation of the S-process at Very Low Metallicity, A \& A, 413, 1073

[14] Truran, J.W., \& Otsuki, K. 2005, in IAU Symp. 228, From Lithium to Uranium: Elemental Tracers of the Early Cosmic Evolution, eds. V.Hill, P. François \& F. Primas, Cosmic Evolution of r-Process Elements, p. 421

[15] Honda, S., et al. 2005, in IAU Symp. 228, From Lithium to Uranium: Elemental Tracers of the Early Cosmic Evolution, eds. V.Hill, P. François \& F. Primas, Subaru HDS Studies of r-Process Elements in Metal-Poor Stars from Near-UV Spectra, p. 429

[16] Cowan, J.J., \& Sneden, C. 2006, Heavy Element Synthesis in the Oldest Stars and the Early Universe, Nature, 440, 1151

[17] Hill, V., et al. 2002, First Stars I - The Extreme r-Element Rich, Iron-Poor Halo Giant CS 31082-001, Implications for the r-Process Site(s) and Radioactive Cosmochronology, $A \&$ \& , 387, 560

[18] Truran, J.W., et al. 2001, Nucleosynthesis Clocks and the Age of the Galaxy, Astrophysical Ages and Time Scales, eds T. von Hippel, C. Simpson, N. Manset, ASP Conference Series, 245, p. 226

[19] Wanajo, S., et al. 2003, The r-Process in Supernova Explosions from the Collapse of O-Ne-Mg Cores, ApJ, 593, 968 
[20] Plez, B., et al. 2004, Lead Abundance in the Uranium Star CS 31082-001, A \& A, 428, L9

[21] Christlieb, N., et al. 2002, A Stellar Relic from the Early Milky Way, Nature, 419, 904

[22] Frebel, A., et al. 2005, Nucleosynthetic Signatures of the First Stars, Nature, 434, 871 